\title{
(Chitosan-g-glycidyl methacrylate)-xanthan hydrogel implant in Wistar rats for spinal cord regeneration
}

DOI:

10.1016/j.msec.2017.03.005

\section{Document Version}

Accepted author manuscript

Link to publication record in Manchester Research Explorer

\section{Citation for published version (APA):}

Elizalde-Peña, E. A., Quintero-Ortega, I. A., Zárate-Triviño, D. G., Nuño-Licona, A., Gough, J., Sanchez, I. C., Medina, D. I., \& Luna-Barcenas, G. (2017). (Chitosan-g-glycidyl methacrylate)-xanthan hydrogel implant in Wistar rats for spinal cord regeneration. Materials Science and Engineering C, 78, 892-900.

https://doi.org/10.1016/j.msec.2017.03.005

\section{Published in:}

Materials Science and Engineering C

\section{Citing this paper}

Please note that where the full-text provided on Manchester Research Explorer is the Author Accepted Manuscript or Proof version this may differ from the final Published version. If citing, it is advised that you check and use the publisher's definitive version.

\section{General rights}

Copyright and moral rights for the publications made accessible in the Research Explorer are retained by the authors and/or other copyright owners and it is a condition of accessing publications that users recognise and abide by the legal requirements associated with these rights.

\section{Takedown policy}

If you believe that this document breaches copyright please refer to the University of Manchester's Takedown Procedures [http://man.ac.uk/04Y6Bo] or contact uml.scholarlycommunications@manchester.ac.uk providing relevant details, so we can investigate your claim.

\section{OPEN ACCESS}




\section{(Chitosan-g-Glycidyl Methacrylate)-Xanthan hydrogel implant in Wistar rats for spinal cord regeneration}

E. A. Elizalde-Peña ${ }^{(1)}$, I.A Quintero-Ortega ${ }^{(2)}$, D.G. Zárate-Triviño( ${ }^{(3)}$, A. Nuño-Licona ${ }^{(4)}$, J. Gough ${ }^{(5)}$, I.C. Sanchez ${ }^{(6)}$, E. Prokhorov $^{(7)}$, D. I. Medina*(8) and G. Luna-Bárcenas*(7)

(1) División de Investigación y Posgrado. Facultad de Ingeniería, Universidad Autónoma de Querétaro, 76010, Santiago de Querétaro, México.

(2) Department of Chemical Engineering, Universidad de Guanajuato, León, GTO México

(3) Department of Biology, UANL, Monterrey, NL México

(4) Instituto Nacional de Rehabilitación, Calz. México Xochimilco No. 289, Col. Arenal de Guadalupe, D. F. 14389, México.

(5) School of Materials: Materials Science Centre, The University of Manchester, Grosvenor Street, Manchester M1 7HS, United Kingdom.

(6) Department of Chemical Engineering, The University of Texas at Austin, Austin, TX 78712

(7)Cinvestav-Querétaro, Libramiento Norponiente No. 2000, Frac. Real de Juriquilla, Querétaro, Qro., 76230, México.

(8) Tecnologico de Monterrey, School of Engineering and Sciences, Atizapan de Zaragoza, Estado de Mexico 52926, Mexico.

\section{*Corresponding authors: dora.medina@cantab.net; gabriel.luna@cinvestav.mx}

Key Words: Hydrogels, Laminectomy, somatosensory evoked potential, degradation, enzyme, lysozyme. 


\begin{abstract}
This work reports the results of in vivo assays of an implant composed of the hydrogel Chitosan-g-Glycidyl Methacrylate-Xanthan $[(\mathrm{CTS}-g-\mathrm{GMA})-\mathrm{X}]$ in Wistar rats. Degradation kinetics of hydrogels was assessed by lysozyme assays. Wistar rats were subjected to laminectomy by cutting the spinal cord with a scalpel. After the surgical procedure, hydrogels were implanted in the injured zone (level T8). Somatosensory evoked potentials (SEPs) obtained by electric stimulation onto periphery nerves were registered in the corresponding central nervous system (CNS) areas. Rats implanted with the biomaterials showed a successful recovery compared with the non-implanted rats after 30 days. Lysozyme, derived from egg whites, was used for in vitro assays. This study serves as the basis for testing the biodegradability of the hydrogels (CTS-g-GMA)$\mathrm{X}$ that is promoted by enzymatic hydrolysis. Hydrogels' hydrolysis was studied via lysozyme kinetics at two $\mathrm{pH}$ values, 5 and 7, under mechanical agitation at $37^{\circ} \mathrm{C}$. Results show that our materials' hydrolysis is slower than pure CTS possibly due to the steric hindrance imposed by the GMA grafting of functionalization. This hydrolysis helps degrade the biomaterial and at the same time it provides support for spinal cord recovery. Combination of these results may prove useful in the use of these hydrogels as scaffolds for cells proliferation and their application as implants in living organisms.
\end{abstract}

\title{
Introduction
}

The spinal cord is elastic, but it is acutely sensitive to direct pressure. Any localized damage to the spinal cord or its roots leads to some degree of paralysis (hemiplegia, paraplegia, or quadriplegia) or loss of feeling in certain parts of the body. Severe damage to the ventral root or ventral horn cells results in the flaccid paralysis of these muscles, which consequently cannot move either voluntarily or involuntarily [1]. The result of an incomplete or complete spinal cord lesion is either paraplegia (paralysis of the lower body) or quadriplegia (paralysis of the body from the neck down), depending on whether the injury was sustained in the thoracic/lumbar region or the neck region of 
the spinal column, respectively [2]. Anyone with a traumatic spinal cord injury (SCI) must be monitored for symptoms of spinal shock - a transient period of functional loss that follows the injury. Spinal shock results in the immediate deficiency of all reflexes, falling blood pressure, and paralysis and insensitivity of all of the muscles below the injury. Neural function usually returns within a few hours after the injury. If function does not resume within 48 hours, paralysis is permanent in most cases [1].

Over the past three decades, there has been encouraging progress in animal models, with sufficient regeneration of the damaged spinal cord to enable some recovery of motor ability. When the spinal cord is injured, the first phase involves mechanical tissue damage. This is followed by a second phase, which implicates the loss of damaged tissue; this is principally caused by a severe local disturbance of the blood supply. [2-4] Attempts have been made to minimize this secondary damage with neuroprotective agents, but so far, only high doses of a synthetic corticosteroid given within the first hours after injury are clinically in use $[4,5]$.

In 2013, the World Health Organization (WHO) estimated that globally, there are about 40 to 80 new cases of spinal cord injury (SCI) per one million people annually, based on quality country-level incidence studies of SCIs from all causes. More specifically, this statistic means that every year, between 250,000 and 500,000 people experience SCI [6].

This information is important to collect since the resource needs and characteristics of traumatic and non-traumatic spinal cord injury (TSCI and NTSCI) populations are different. Historically, up to $90 \%$ of SCI has been traumatic in origin, but data from the most recent studies indicate a slight trend in recent years towards an increase in cases of NTSCI [6].

Based upon available evidence on the etiology of TSCI across WHO regions, the three most common causes of TSCI are transportation accidents (road traffic crashes in particular), falls and violence. Meanwhile, the leading causes of NTSCI are neoplastic tumors and degenerative conditions of the spinal column, followed by vascular and autoimmune disorders. Congenitally and genetically caused cases such as spina bifida are not recorded, since these are typically collected in different settings [6]. 
Country-level TSCI incidence rates vary widely across the world (13 to 53 cases per million population). TSCI incidence rates tend to be higher in North America than in Europe, possibly due to the higher rates of violence in the U.S. TSCI incidence data from other regions either do not exist, or they fluctuate too widely among and within countries that it is difficult to provide valid summary statistics. Since NTSCI is more common in older age groups, and given global aging, NTSCI incidences may increase and overtake TSCI incidences within the next few decades [6, 7]. Finally, concerning both TSCI and NTSCI, incidence rates are higher among males than females.

SCIs may involve damage to a substantial amount of tissue, including the white and gray matter as well as blood vessels. This occurs either following trauma; a degenerative process; suffering a stroke, where the amount of tissue damage may increase with secondary pathophysiological changes; or following surgery, where sectioning neural tissue is unavoidable during elective oncological surgery, which necessitates the removal of a rim of vital tissue from around a tumor [8].

Whilst the functional deficit is related to the amount of tissue damage and to the interruption of the associated axonal pathways, it is also related to the inability of the adult mammalian CNS to repair its own structures and to restore the damaged tissue, which occurs in the CNSs of adult inframammalian vertebrates. Tissue repair results in scarring, despite evidence that the CNS has significant repair potential following injury and would be capable of restoring a cellular field for axonal growth [8,9].

Recovery of the spinal cord is complicated, as it receives sensory information from almost all of the tissues in the body. It transmits this information in the form of electrical impulses to the brain along millions of nerve fibers that are grouped together in bundles. A sharp blow to the spinal column can cause dislocation of individual vertebrae and severe damage to the spinal cord, including its complete severance [2].

Life expectancy is defined as the average remaining years of life for an individual. Life expectancies for those with SCIs continue to increase, but they are still somewhat below the life expectancies for those without SCIs. Mortality rates are significantly higher during the first year after injury than during subsequent years, particularly for severely injured persons [7].

Because of the aforementioned factors, it is important to use biological models to 
simulate the environment of the human body. The present study continues the trend of using Wistar rats in order to observe the implant's behavior in vitro when it comes into contact with enzymes such as lysozyme, a natural defense in the human body.

Lysozymes, also called muramidases, are present in easily detectable amounts in human breast milk, tears, saliva, and serum; they are considered to be self-defense enzymes. Lysozymes have been classified into three distinct forms by their amino acid sequences and tertiary structures [10-12].

Lysozymes have a low level of endochitinolytic activity, but they can be distinguished from chitinases because they readily hydrolyze. However, some studies have reported using lysozymes to determine the biodegradation of some CTS derivatives - the modification of the degree of acetylation provides a powerful means for controlling biodegradation and biocompatibility, and it can be optimized for tissue engineering applications, increasing the importance of this enzyme [13, 14].

The present work attempts to determine the activity of the (CTS-g-GMA)-X as an implant in Wistar rats by means of somatosensory evoked potentials (SEPs) measurements to assess its potential as smart biomaterial. It also investigates the enzymatic degradation's susceptibility and hydrolysis of the biomaterials when contacted with lysozyme in vitro.

\section{Materials}

Commercial reagents used in the present study were obtained from Sigma-Aldrich Chemicals; they did not undergo additional purification. The X used in this work is foodgrade and has an average molecular weight of $4.96 \mathrm{kDa}$, determined by capillary viscosimetry (Ostwald viscometer) in water at a constant temperature of $25^{\circ} \mathrm{C}$. The CTS has an $87 \%$ deacetylation degree, determined via titration, and it has an average molecular weight of $300 \mathrm{kDa}$, determined by viscosimetry in a $0.1 \mathrm{M}$ acetic acid and 0.2 $\mathrm{M}$ sodium chloride solution at $25^{\circ} \mathrm{C}$. The Mark-Houwink constants, $\alpha$ and $\mathrm{K}$, were taken from Milas et al. for the X and from Kassai et al. for the CTS [15, 16]. The GMA liquid is $97 \%$ pure and was used without additional purification. 


\section{(CTS-g-GMA-X) Hydrogel Synthesis}

The (CTS-g-GMA)-X hydrogels were synthesized as described by Elizalde-Peña et al. 2013[17]. The method was modified in two different ways by incorporating neutral and acidic aqueous media. The (CTS-g-GMA)-X hydrogels were labeled as Z11E, Z12E, $\mathrm{Z} 13 E$, and $\mathrm{Z} 14 E$, respectively. The numbers $1: 1,1: 2,1: 3$, and 1:4 correspond to stoichiometric molar ratios of CTS:GMA. $E=$ A for CTS-g-GMA materials dissolved in the acid solution and $E=\mathrm{B}$ for those dissolved in the neutral solution [17].

\section{Water Vapor Permeability}

A film was placed in a permeability chamber and then hermetically sealed with Teflon. The chamber was placed in a desiccator with a vacuum; silica was used as the secant agent. The interior maintained an average relative humidity of $10-12 \%$.

The chamber was maintained at a constant room temperature and weighed each hour until it reached equilibrium. The chamber contained distilled water, providing a relative humidity of $100 \%$. The permeability was obtained using the gravimetric method described in ASTM E96 [18].

\section{Hydrogel Pre-Treatment for Implants}

Hydrogels were immersed in 70\% ethanol for 20 minutes and then further dried out in a laminar flow hood for 16 hours. Then, they were placed in 24-well plates and sterilized using Sterrad equipment (Johnson \& Johnson) [19, 20].

\section{Implants in Live Organisms}

Male Wistar rats with an average weight of $300 \mathrm{~g}$ were used in the present study. A laminectomy was carried out on the rats at vertebral body T8 to leave the spinal cord exposed and to injure it by means of a cut via bistoury. Then, the polymer was placed on 
the injured zone (Group 1) and the incision was closed. Group 2 was used as a control, undergoing the same spinal cord injury but without the polymer implant.

Previous to the injury, left sciatic nerve dissection was performed to stimulate it by means of hook electrodes via a silver chloride wire connected to the stimulation system of the registry equipment (Cadwell 5200 A) for SEPs. The registry electrodes were in the form of silver disks measuring $4 \mathrm{~mm}$ in diameter. They were placed on the skin that covers the skull over both parietals. The earth electrode was fixed to the tail and the SEPs were obtained with the following parameters: gain (20 microvolt by division), filter of discharges (500 Hertz), filter of losses (10 Hertz), and scan speed (5 ms). The average registry obtained was 200 signals from a stimulus of 0.5 volts of intensity and 0.2 ms of duration with $0.5 \mathrm{~Hz}$ of frequency. The same procedure was conducted after the injury to the rats' spinal cords.

SEP measurements were obtained by means of the previously described technique 5,15 , and 30 days after injury. In each group, after obtaining the SEP, the animals were sacrificed by means of an anesthesia overdose. Their spinal cords were retained for later histological analysis.

Figure 1 illustrates the procedure used to determine the SEPs of the rats and the physical location of the electrodes. Surgical, aseptic and bioethics cares were taken during the surgical procedure.

\section{Enzymatic Activity of the Lysozyme}

Enzymatic activity was determined using $p$-Nitrophenol-N-acetyl- $\beta$-Dglucosamine as a substrate, which was added to a buffer with a $\mathrm{pH}$ of 5.6, distilled water, and the sample. The temperature was $37^{\circ} \mathrm{C}$ and the samples underwent constant agitation for 1 hour. A spectrophotometer was adjusted for the measurements with a $400 \mathrm{~nm}$ wavelength [21].

\section{Kinetics of Hydrolysis for Biopolymers via the Lysozyme}


Kinetics was performed with buffer solutions with $\mathrm{pH}$ values of 5.0 and 7.0. The assay was created under sterile conditions by adding the following to an Erlenmeyer flask: $0.1 \mathrm{~g}$, dry weight, of biomaterial to the buffer solution after 43,600 units of lysozyme per $\mathrm{mL}$ was added. This solution was agitated in an orbital shaker at 200 rpm maintained at $37^{\circ} \mathrm{C}$ for 7 days; $5 \mathrm{~mL}$ aliquots were obtained daily. The aliquots were centrifuged at 10,000 rpm for 10 minutes to determine the total and reduced sugars.

\section{3, 5-Dinitrosalicylic Acid (DNS) Technique}

$0.25 \mathrm{~mL}$ of DNS reactive were added to $0.25 \mathrm{~mL}$ of the sample. This mixture was warmed in a boiling water bath for 5 minutes and was then cooled in an ice bath for 3 minutes in order for finish the reaction. Finally, $2.5 \mathrm{~mL}$ of distilled water was added. The blank was made by replacing the sample volume with distilled water; the pattern curve was created using glucose. This technique was performed in a spectrophotometer with a wavelength of $540 \mathrm{~nm}[22]$.

\section{Results}

Water Vapor Permeability

Table 1 shows the permeability values for hydrogel $\mathrm{Z}$, series A and B. The values obtained show a low permeability in both cases; this is in comparison with the polymers, such as polyester and beeswax, as well as several chitosan-based polymers in CTS. This indicates that these polymers are a good barrier for preventing continuous water vapor flow [23-26].

Table 2 shows different polymer permeability values, which can be compared to the highest and lowest values of our synthesized materials. The results reveal that our biomaterials provide a better barrier than some of the polymers of natural or synthetic origin [23-26].

These findings indicate that both series of materials, A and B, could be considered for use as cell scaffolds or implants in tissue engineering, as these materials allow for the 
flow of small quantities of water vapor. It is expected that they would allow for the flow of some metabolites when implanted in living organisms.

\section{Implants in Wistar Rats}

Figure 2 shows the surgical procedure for extracting the rats' spinal cords. All of the surgeries to injure the rats' spinal cords as well as all of the surgeries to remove their spinal cords post-treatment were successful.

The red arrow in Figure 2 marks the injury site in the rats and the site of the spinal cord implant. The implant size is $5 \times 5 \mathrm{~mm}$ due to the injury, and the location was in a layer of biomaterial. This represents a significant advance in the current literature, since the polymer showed no visible degradation, no severe damage to the spinal cord structure, and no apparent immunological rejection. These findings support the use of polymers as an implant in live organisms, serving as a third-generation material.

Figure 3 shows SEP measurements in which the frequency of component losses recorded after injury is clearly evident. The existence of components with a correct definition in the measurement screen can be seen in the upper channels, i.e., channel 1 in Figure $3 a$ and channels 1 and 2 in Figure $3 b$.

In Figure 3a, channel 2, the signal is an almost straight line after injury, indicating that the rat lost the movement of its back legs and all sensation in this part of its body owing to the nonexistence of stimuli through the spinal cord. Similar behavior can be observed in Figure 3b, channels 3 and 4.

Figures $3 a$ and $3 b$ show SEP registers from two different rats; these are considered representative results for all tested specimens. Laminectomy was performed by the same procedure in both rats. Figure $3 a$ shows channels 1 and 2 and Figure $3 b$ shows channels 1-4. This is because these readings were taken at different spots in the two different rats. The rats whose results are shown in Figure $3 b$ were tested (SEP registers) at two spots instead of one (Figure 3a). These graphs show evidence of the loss of components in the SEP after laminectomy. This behavioral trend was the same as that presented in each rat. 
Because it is possible to observe the SEP registers in both groups (groups 1 and 2) before the laminectomy (Figure 3a, channel 1, and Figure 3b, channels 1 and 2), negative and positive components are shown between 15 and $25 \mathrm{~ms}$ of lag time; these components disappear after laminectomy (the rest of the channels).

SEP obtained 30 days after the surgery in group 1 again shows the presence of components, in which the negative component between 15 and $25 \mathrm{~ms}$ of lag time is always present in both groups. For group 2, the results show an unstable basal line, and it is not possible to identify components in the SEP.

Five days after surgery, it is possible to observe a recovery in the components of the Group 1 SEPs. In addition, these rats show physical improvement compared to Group 2 (Figure 4).

Figure 4 shows four rats of group 1 with an implant; in all of them, the sciatic nerve and right hind leg (hindquarters of the rats showed loss of movement) were measured. Each figure shows a different rat, and all show very similar behavior. The data collected for the measurements of the four channels of group 1 of the rats is five days after the laminectomy.

In all of them, there is evidence that the presence of negative and positive components between 15 and $25 \mathrm{~ms}$ of lag time are weak compared with the components before injury. However, it is clear the rats recovered slightly, a fact supported by their physical recovery, in which the rats had partial mobility of their hindquarters, albeit without control.

Figure 5 shows the most representative plot of group 2 (without the implant) after 30 days. These rats show the same behavior. The rats of group 2 without the polymer implant show an unstable basal line. It is not possible to clearly identify any component for them (Figure 5). The opposite occurs for group 1, with the polymer implant, where again the presence of components is shown, as is possible to observe in Figure 6, in which the negative and positive component between 15 and $25 \mathrm{~ms}$ of lag time is always present in both figures. The rats of group 1 (with the implant) at 30 days show favorable recovery; however, they do not show full recovery, and are able to move their hindquarters in only an uncoordinated manner. 
In order to compare the recovery of rats, SEPS measurements are shown in Figure 7, where it is possible to demonstrate that group 1 recovers almost the original behavior in SEP; additionally, group 1 practically showed a recovery of up to $90 \%$ according to the Wrathal test, while that of group 2 was only around $70 \%$ [27].

Thirty days after laminectomy, it is not possible to identify any SEP components in Group 2 because they are not resolved (Figure 5); furthermore, the rats do not display any movement or signals of recovery.

Meanwhile, for Group 1 (Figure 6), the SEPs obtained 30 days after surgery can be compared with the potential observed in the upper channel of Figures $3 \mathrm{a}$ and $3 \mathrm{~b}$, which indicates a satisfactory recovery of the injury. In addition to the recovery of the SEP components, the rats could move the back part of their body without any signs of damage caused by the laminectomy. This effect in the recovery of the rats can be attributed to the presence of the polymer in the injured zone.

SEP results are listed together for the comparison in Figure 7. All channels and components are clearly shown on it. Figure 7 shows progressive SEP data of rats across the experiment: a) before and after laminectomy, b) Group 2, 30 days after surgery, c) and d) Group 1 after 30 days.

A previous study demonstrated that hydrogels were suitable for the preservation of cells NG108-15, which was made through a DNA assay (not presented in this article); a Neurofilament $\mathrm{H}$ stain reveals that three polymers of series $\mathrm{Z}$ allow the differentiation of a small number of cells. Figure 8 shows the hydrogels Z series A (Z11A and Z14A) and reveals that the representative cells differentiated to neurites in red.

Figure $9 \mathrm{~b}$ shows the hydrolysis kinetics for CTS by the lysozyme at a $\mathrm{pH}$ of 7 . It is possible to observe the behavior of the sample and the control: until 100 hours, they do not show hydrolysis, after which point they begin hydrolysis at the same rate for the next 20 hours. Nevertheless, after more time, the hydrolysis percentage maintains a value of $1.12 \%$; meanwhile, the sample values increase over time. The highest value for the sample is $2.8 \%$ at 168 hours.

Figure 9a shows the hydrolysis kinetics for $\mathrm{CTS}$ at a $\mathrm{pH}$ of 5 . In this case, the hydrolysis percentage is high compared to the sample at a $\mathrm{pH}$ of 7 . This behavior could be attributed to the great affinity of CTS for polar solvents in acidic mediums [28]. This 
suggests the relaxation in the chain of the backbone of the CTS, which is how the lysozyme can hydrolyze the material in an acidic $\mathrm{pH}$ more easily than in a neutral $\mathrm{pH}$ more. In addition, CTS at a pH of 5 shows the lowest start time of hydrolysis (48 hours). This can be compared with similar polymers reported in literature, where the optimal $\mathrm{pH}$ and temperature for the lysozyme were 5 and $37^{\circ} \mathrm{C}$, respectively [29].

The solubility of CTS is mainly related to an acidic $\mathrm{pH}$, as it allows for the protonation of the amino groups of the molecular structure of CTS, excluding intermolecular hydrogen bridges and causing the polymer's solubility. [28] This suggests that CTS and the hybrid CTS-g-GMA could be dissolved or re-dispersed in the media used in this assay, allowing the enzyme to hydrolyze the materials with great efficiency. This effect can be observed in Figure 10a, which shows the hydrolysis kinetic of CTS- $g$ GMA, where the start time of hydrolysis is 72 hours and the maximum percentage of hydrolysis is $4.77 \%$.

Figure 10a shows the hydrolysis kinetics for CTS at pH of 5. In this case, the hydrolysis percentage is high compared to the sample with $p H$ of 7. This behavior could be attributed to the great affinity of CTS for polar solvents in acidic mediums [28]. This suggests relaxation in the chain of the backbone of the CTS, which is how the lysozyme can hydrolyze the material under acidic pH more easily than under neutral $p H$. In addition, CTS at pH of 5 shows the lowest start time of hydrolysis (48 h). This can be compared with similar polymers reported in literature, where the optimal pH and temperature for the lysozyme were 5 and $37^{\circ} \mathrm{C}$, respectively [29].

CTS-g-GMA at pH 5 showed two times the percentage of hydrolysis compared with the sample at pH 7 (Figure 10b). The solubility of CTS is mainly related to the acidic pH, as it allows for the protonation of the amino groups in the molecular structure of CTS, which excludes intermolecular hydrogen bridges and causes the polymer's solubility [28]. Figure $10 \mathrm{~b}$ shows the major percentage of hydrolysis for the control sample at intermediate times; at $168 \mathrm{~h}$, the CTS-g-GMA exceeds the control hydrolysis. This suggests that the CTS and hybrid CTS-g-GMA could be dissolved or redispersed in the acid media used in this assay, allowing the enzyme to hydrolyze the materials with great efficiency. 
Furthermore, these results demonstrate that the hybrid CTS-g-GMA is more stable compared with CTS mainly at pH 5.

All the evaluated materials, namely, CTS, CTS-g-GMA, and (CTS-g-GMA)-x, showed similar behavior and showed the mayor percentage of hydrolysis at $\mathrm{pH} 5$. These results can be explained by the combination of the optimal operation $\mathrm{pH}$ of lysozymes and the solubility of CTS in acidic media. Biological fluids with $\mathrm{pH} 7$ allowed the stability of the materials after $168 \mathrm{~h}$ and realized $3.5 \%, 2.5 \%$, and $3.2 \%$ hydrolysis.

The hydrogel (CTS-g-GMA)-X behaves differently than the other materials, as the bigger enzymatic activity occurred at 72 hours for the materials to reach a $\mathrm{pH}$ of 5 and at 120 hours for the materials to reach a $\mathrm{pH}$ of 7 (Figures 11a and 11b, respectively). There was no notable difference between the treatments; however, the hydrogel at a $\mathrm{pH}$ of 7 showed higher values for the percentage of hydrolysis than it did at a $\mathrm{pH}$ of 5 . Although the optimal enzymatic $\mathrm{pH}$ is 5.5 , the difference may be due to the $\mathrm{X}$, which is soluble at neutral and has a slight alkalinity media [30].

\section{Conclusion}

Low values of permeability obtained from the biopolymers indicate that the biomaterials used in the present study provide a better barrier for allowing a small flow of water vapor when compared to several natural polymers (including some CTS-based). The implantation of hydrogel Z (series A and B) in the spinal cords of Wistar rats after laminectomy promoted their recovery 30 days after surgery, which is about one-fifth of the normal recovery time. The percentage of hydrolysis, obtained from the kinetics at different $\mathrm{pH}$ values showed that the materials could be degraded by tissular fluid; hydrolysis after 120 hours is $c a$. $5 \%$. This hydrolysis helps degrade the biomaterial and at the same time it provides support for spinal cord recovery. The conjunction of these results are promising and strongly support the future use of these hydrogels as scaffolds for cells proliferation as well as their application as implants in living organisms. 


\section{Acknowledgements:}

The authors would like to thank CONACYT Mexico for the financial support given this

project (Grant Number 181678). We thank Efren Alatorre for the support in SEP measurements. 